\title{
Two discrimination test apparatuses for primates*
}

\author{
D. M. RIMBAUGH, C. L. BELL, and T. V. GILL \\ Yerkes Regional Primate Research Center, Atlanta, Georgia 30322 \\ and \\ Georgia State University, Atlanta, Georgia 30303
}

This paper describes two test apparatuses developed to facilitate discrimination learning studies with primates. The semiautomated apparatus provides for presentation of stereometric stimulus materials through the illumination of lamps which make a one-way mirror transparent at the onset of a trial. The totally automated test system was developed to facilitate the collection of Transfer Index (TI) measurements, measurements intended to provide for equitable assessments of the learning-set capabilities of diverse primate genera. The TI test procedures and the system's operations provide for criterional mastery $(67 \%$ or $84 \%$ responses correct) of two-choice visual discrimination problems, consisting of projected patterns, then reversal of cues for the 10 test trials that are of particular significance in calculation of the $\mathrm{TI}$ scores.

Davenport, Chamove, and Harlow (1970) have recently described a semiautomatic version of the widely used Wisconsin General Test Apparatus (WGTA) that affords E many procedural options not provided for in the original versions (Harlow \& Bromer, 1938; Harlow, 1949). That revision consisted fundamentally of elaborations upon the basic components of the WGTA, such as provision for controlled lowering and raising of a modified forward screen and adaptation of the stimulus tray to allow $\mathbf{E}$ options in baiting procedures and to obtain S's response latency per trial.

The first portion of the present paper describes still another semiautomatic WGTA; the second portion describes a totally automated test unit designed to facilitate collection of Transfer Index (TI) measurements (Rumbaugh, 1970). Both units can be adapted for use in a wide variety of discrimination learning studies with diverse primate forms, including children.

\section{DESCRIPTION OF THE \\ SEMIAUTOMATED APPARATUS Frontispiece}

The frontispiece (Fig. 1) is designed to attach directly to S's home or restraint cage. The discriminanda,

*This work was supported by NIH Grant RR-00165 and NSF Grants GB-7161, GB-19146, and GB-11850. The authors gratefully acknowledge the work of Mr. Carl Spears, an electronics technician at San Diego State College, who contributed significantly to early versions of the apparatuses herein described. placed on a ledge behind mirrored glass as discussed later in this paper, become visible to $S$ at the onset of a given trial through the illumination of bulbs positioned over the objects. A choice consists of reaching toward one of the two stimuli, and as S's hand (or nose) comes to within $1 / 2$ in. of the glass, a beam of light is broken. Instantaneously the lights that illuminate the objects for S's viewing are turned off, causing the objects to disappear and ending the trial. Correct choices are rewarded with sucrose or an M\&M candy, dispensed from a Davis MMD-2 unit, and an associated tone that might serve as an immediate conditioned reinforcer. It should be noted that the response required entails only minimal energy expenditure and should not differentially fatigue diverse primate forms.

The frontispiece is made in three sections. On E's side of the sheet metal that forms the door of S's cage is a piece of wood 1 in. thick with an aperture, $23 / 4 \times 111 / 4$ in., that forms the window through which $S$ views the problem. The photocells and light sources are located, respectively, in the upper and lower ledges of this window so as to be near the midline of each discriminandum. To protect the photocells and the wood, the window facing is lined with 1/8-in. Plexiglas.

Immediately behind the wooden piece is a second one that has an aperture, $3 \% \times 12 \frac{1}{4}$ in., somewhat larger than the aperture of the first wooden piece. This second piece surrounds and holds the glass

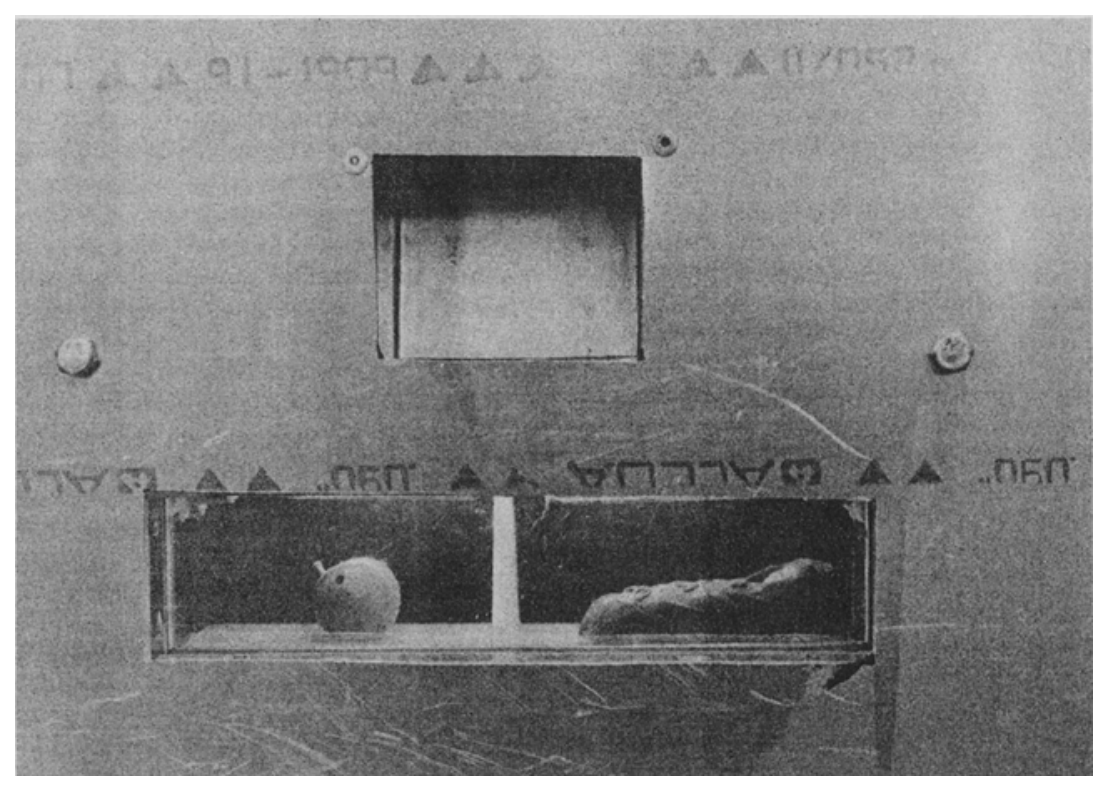

Fig. 1. S's view of frontispiece with trial simulated. M\&M candy rewards are automatically dispensed into the receptacle above the discriminanda. (The projection screens of the automated test unit described later in this paper are positioned where the objects are seen in this figure.) 


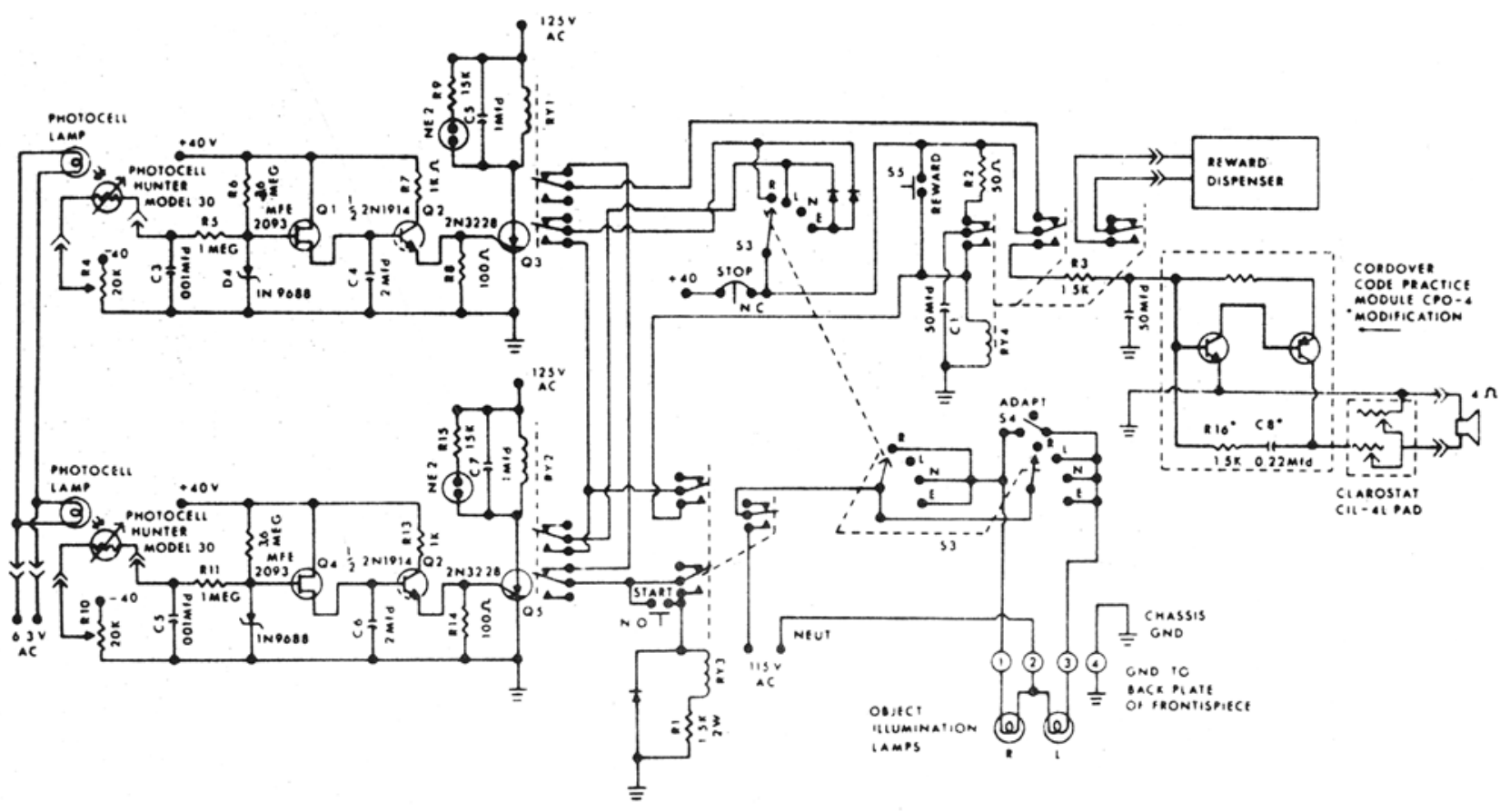

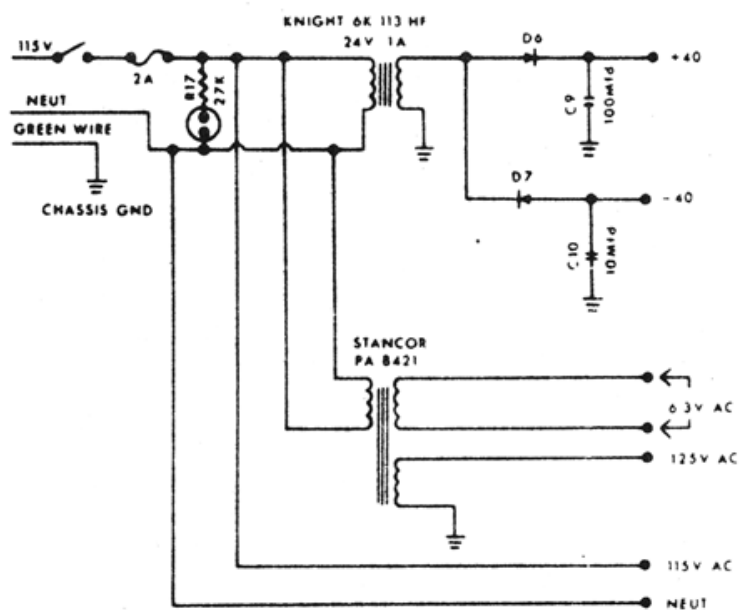

Fig. 2. Circuitry of the control unit.

described below, while the first piece frames the glass, securing it in at the front. This piece was planed to a thickness of 25/32 in., which is $1 / 32$ in. thicker than the total thickness of the glass, so that all pressure is exerted upon the wood when the assembly bolts are tightened.

Three pieces of glass are used in the window. Closest to $S$ is a $1 / 4-$ in. thickness of laminated safety glass used to provide a strong and essentially scratch-proof surface to withstand S's fingernails. The next or middle piece of glass is a $1 / 4-\mathrm{in}$. piece of "one-way mirror" or "transparent mirror" to block the S's view of the discriminanda and E's placement of them between trials; it becomes transparent when at the onset of a trial the lamps are activated. The most remote piece of glass consists of Lexan, a material similar to Plexiglas in appearance but stronger.

The back section, made of $1 / 4$-in. aluminum alloy, has a $2 \frac{1 / 2}{2} 11$ in. aperture and serves as the rear frame for the glass sections. The frontispiece so constructed is tough enough to withstand the indulgences of all apes as well as other primate forms.

\section{Photocell Amplifier}

The photocell voltages are amplified by a dual-channel three-stage solid-state amplifier (see Fig. 2 for the basic circuit).

\section{Control Circuit ${ }^{1}$}

Most of the remaining wiring is for optional modes of operation used only for shaping choice behaviors in naive Ss, a matter discussed later.

Before starting the trial, E positions the discriminanda and then with reference to their + and - cue valences sets the right-left switch of the control unit to determine which one of the relays in the photocell circuits (Relays 1 and 2) will, if energized during the response, pull in Relay 4. Relay 4, when energized, causes the reward to be dispensed and the tone to be sounded. To initiate a trial, E presses the START button, energizing Relay 3, which latches itself in until the circuit is broken by the opening of a set of normally closed contacts or Relay 1 or Relay 2, one of which pulls in momentarily for each response. 


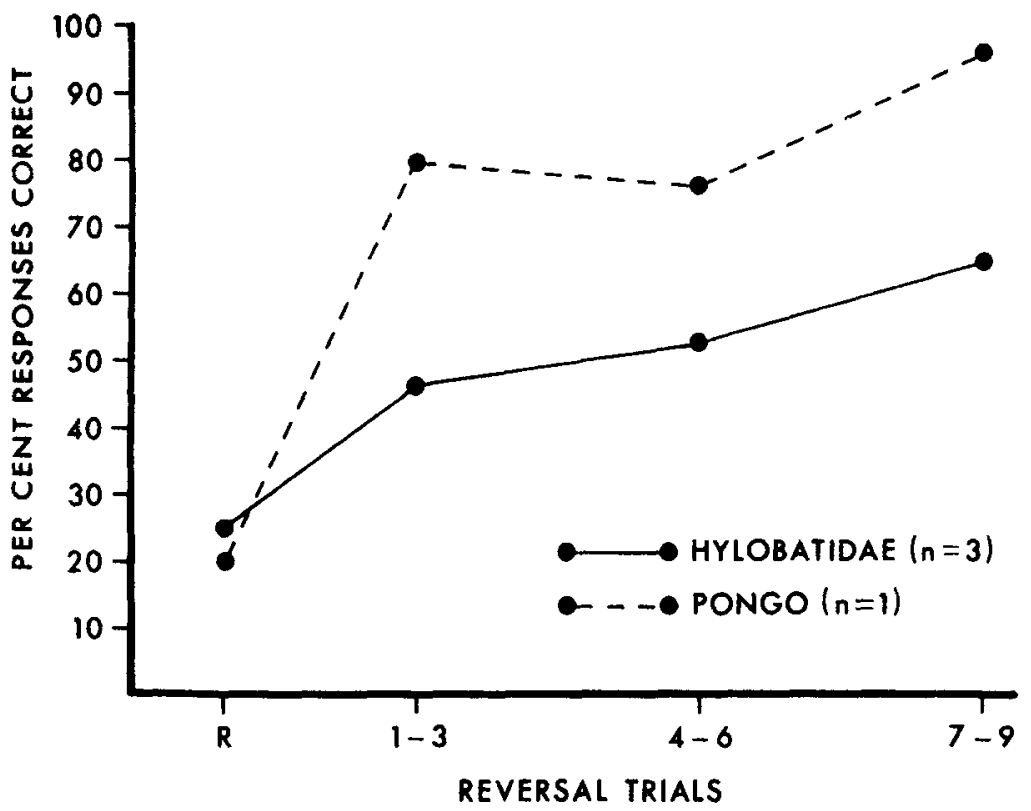

Fig. 3. Reversal performances from 20 problems for Ss as keyed. Prior to cue reversal, problems were mastered to the criterion of $84 \%$ choices correct according to a graduated criterion (Table 1). On the average, Ss required from 15 to 20 trials to achieve criterion, the occasion for cue reversal.

Additional contacts of Relay 3 complete the lamp circuit and enable Relay 4 to be pulled in if the response is correct. Relay 4 is disabled during the time between trials to prevent $S$ from gaining spurious rewards by making responses between trials. One set of contacts of Relay 4 is used as a "pulse stretcher" capacitor (C1) to provide timing for the tone. The 47-ohm resistor (R2) in the charging circuit is required to prevent contact damage due to the sparking that would occur if the discharge capacitor were choice behaviors of naive Ss, a few additions were indicated. connected directly to the $40-\mathrm{V}$ source by Relay 4.

\section{Shaping Modes}

For normal operation with trained animals, the circuits already described would be sufficient. In order to use the equipment for shaping choice behaviors of naive $\mathrm{Ss}$, a few additions were indicated.

Relay 4 is wired so that it will, when energized, drop out Relay 3 . Thus $\mathrm{E}$ can completely simulate the consequences of a correct response by pressing a REWARD button which simply energizes Relay 4 . This feature facilitates shaping the behaviors of naive Ss for testing.

In the ADAPT mode, assigned by a position of a toggle switch (S4), a light shines only over the correct object, whereas in the test mode both lamps shine. Two additional positions were added to the RIGHT-LEFT switch (S3) so as to allow response to EITHER or NEITHER position to be are potentially subject to alteration (color and brightness) within the course of a given trial through use of appropriate filters interposed between the light source and the stimulus objects. Finally, E is spared many tedious duties, such as removing food from wells after $S$ makes an error, retrieving objects taken by $S$ from a stimulus tray, etc.

\section{DESCRIPTION OF THE AUTOMATED APPARATUS}

Many attempts have been made by various researchers to perfect automated test apparatuses for studies of primates' visual discrimination performances in general and their learning set (Harlow, 1949) capabilities in particular. Characteristically these attempts have left $E$ with copious amounts of frustration and $S$ with little other than low-level performances. Meyer, Treichler, and Meyer (1965) have reviewed the major efforts to develop such systems and concluded in reference to the WGTA (Harlow \& Bromer, 1938; Harlow, 1949), "Though the manual method, in this day of the machine, is pitifully oldfashioned, it has set a standard of efficiency that hasn't been approached by any other apparatus yet [p. 45]."

The first author has reported (Rumbaugh, 1969) the development of a learning-set relevant measurement, termed the Transfer Index (TI), intended to provide for more equitable conduct of cross-species studies of complex learning capabilities than is possible through the use of Harlow's basic fixed-trial learning-set testing methods. The TI assessment procedures focus upon discrimination-reversal performances subsequent to criterional acquisition of each of a series of two-choice problems.

Discussion of the merits and methods of TI testing has been presented elsewhere (Rumbaugh, 1970). Only one of its fundamental characteristics-criterional mastery on each and every problem of a series prior to cue reversal-needs to be reemphasized in the present paper, for it is likely the critical factor in the determination of success with the automated system described herein. Criterional mastery of each problem is believed to sustain the basic processes of attention and discrimination learning that are otherwise not likely sustained in a t o mated multiple-problem test situations if only a relatively small and fixed number of trials are given per problem.

The automated TI testing system, described below, builds upon the basic components of the semiautomated apparatus described above. 
THE AUTOMATED TI SYSTEM ${ }^{2}$

The system comprises the frontispiece and a control unit that contains all of the circuitry for automated programming of trials and recording the events of each trial-the right-left placement of the correct stimulus, S's choice, whether the trial was one of criterional acquisition training or one of reversal testing, and latency of response in seconds.

\section{Frontispiece}

The automated TI system uses the same frontispiece used in the semiautomated one, except for the deletion of the mirror and the substitution in its stead of a piece of Lexan or Plexiglas and the placement of a pair of single-plane readout projectors where the three-dimensional discriminanda were positioned for each trial. The basic projector (Industrial Electronic Engineer, Inc., Van Nuys, California; Model IEE $360 \mathrm{H}$ ) has been modified by cutting a slot on the topside of the outer casing to allow ready extraction of the lens pair which encases the filmed material to be projected. (A finger hole in the bottom of the casing facilitates lifting of the lenses for final extraction.) While this type of projector has its deficiencies with regard to both sharpness of focus and center of focus per lens, it has proven quite satisfactory for our purpose.

As each projector provides for the independent projection of 12 separate images, with identical film materials in a pair of projectors, one has six pairs of images (problems); random right-left assignment of position of the discriminanda across the trials of a problem can be achieved in this manner to render a position unreliable as a cue.

We have used cutouts of magazine advertisements as a prime source of varied yet highly discriminable patterns; these magazine materials serve the same purpose as the refuse o bject used as discriminanda in conventional WGTA testing of learning-set skills. We have also perfected photographic and film cutting techniques that allow for precise duplication of materials, a critical factor for use of a pair of projectors. The precise cutting of duplicate films, each containing 12 patterns in a $3 \times 4$ matrix, is accomplished through use of surgical blades and a template secured on a back-lighted cutting table. A library of several hundred problems has been developed for multiple-problem testing as required both by learning-set and TI methods. 3

Note should be made of the positions of the photocells on the frontispiece which require S's hand to

Table 1

$67 \%$ Level*

$7,8,9,10$, or 11 of the first 11 trials

9 out of the first 14 trials

10 out of the first 16 trials

12 out of the first 19 trials

14 out of the last 21 trials

$* 7$ out of $11=63.5 \% ; 8$ out of $11=72.7 \%$

The investigator may alternate these two levels by means of a switch so that alternate problems are taken at 7 out of the first 11 trials if criterion is reached at that point and the others at 8 out of 11 if criterion is reached at that point. This is done to approximate the desired $67 \%$ level. Although the equipment will reverse at 9,10 , or 11 out of the first 11 trials, reversal is not permitted in $T I$ testing.

tAn explanation similar to that above also applies to the case of 17 or 18 out of 21 . They are alternated to approximate $84 \%$. It is also the case that if a score of 10 or 11 out of the first 11 trials should occur, reversal is not permitted.

come within at least $1 / 2$ in. of a projector's screen, a point that ensures stimulus-contiguous responses (Meyer, Treichler, \& Meyer, 1965). Since the screen is not displaced by S's response, the image remains in focus. As stated above, correct responses are appropriately rewarded, but in any event, a choice results in the turning off of the projectors and the termination of a trial. Whereas the intertrial time is adjustable, we have held it as a constant ( $2 \mathrm{sec}$ ) to date. The system also requires $S$ to withdraw from the screens (to keep the light beams unbroken) for at least $2 \mathrm{sec}$; failure to do so results in a continuing delay of the next trial. This contingency prevents $S$ from executing specious choices upon the instant of trial presentation, thereby continuously cycling the system.

In accordance with TI testing procedures, the system is programmed to allow for either $67 \%$ or $84 \%$ criterional mastery prior to cue reversal (Table 1). (If desired, the system also will sustain initial cue value assignments indefinitely.) Upon achievement of the criterion, the cue values are reversed; upon completion of the 10 th reversal trial, the system is deactivated. The $E$ then advances the system to the next problem through use of two rotary switches, whereupon criterional training is again resumed. If mastery is not achieved within 60 trials, the system also is deactivated. Finally, the system allows for the option of successive criterional reversals with a single problem should E so desire.

Returning to the matter of criterional achievement, it should be noted in Table 1 that beyond the 21st trial and to the 60th trial of a given problem, only the last 21 trials are used for determination of criterional achievement. Thus, a 21-position memory suffices for monitoring and programming.

\section{Control Unit}

The control unit consists of the following components: (1) a system of
21 memory cells for monitoring the correctness of S's choices in reference to the criterion schedules; (2) a Moduprint counterprinter (Column 1 prints " 0 " for errors and " 1 " for correct choices, Column 2 prints " 0 " for criterional training and " 1 " for reversal test trials, Column 3 prints " 0 " and " 1 ," respectively, for the right-left position assignments of the initially correct stimulus, and Columns 4-6 record latencies in seconds); (3) a pair of rotary switches for $E$ to select from the six problems contained within each of the two projectors; (4) a "standby" switch that controls activation of the system; (5) sensitivity controls for the photocells of the frontispiece; and (6) various other controls that allow for simulation of a correct trial, dispensation of a reward, deletion of the tone, brightness of the incorrect stimulus (to allow "fading-in" of it if desired during shaping), etc.

\section{Memory System}

The test begins with all 21 memory cells in the " 0 " state. For the first 21 trials, the 21 individual cells are scanned in sequence, a " 1 " being placed in a respective cell if the response on that particular trial is correct. Two counters are also involved in the test apparatus, one to count the total number of trials and the other to count the total number of correct trials. The circuit effects a reversal if and only if the combination of total trials and correct trials is one of the combination shown in Table 1.

If criterion has not been reached within the first 21 trials, the 21 memory cells (still containing the information from the first scanning) are readdressed in sequence during subsequent trials. However, after the 21st trial, only the correct trials counter is used in determining if the desired criterional level has been reached (by continuing to count up when a " 0 " is replaced by " 1 " and counting down one whenever a " 1 " is replaced by a " 0 " in one of the memory cells). In this manner the 


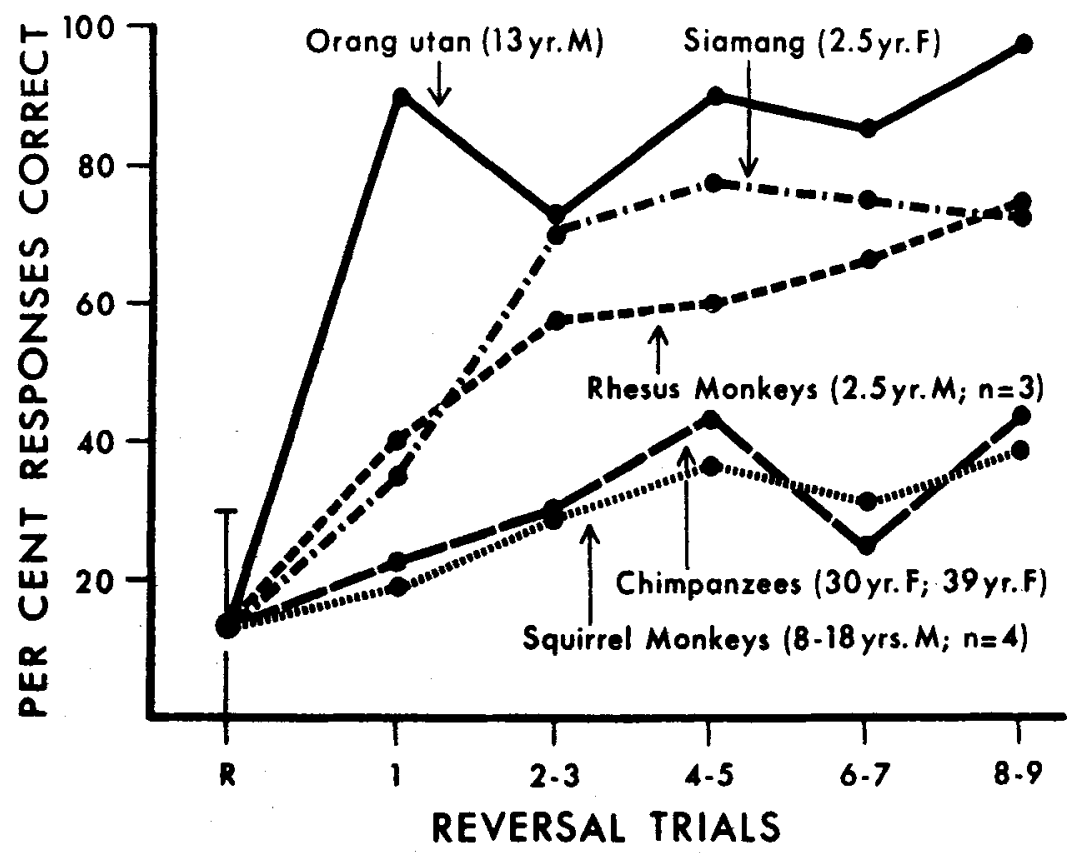

Fig. 4. Performance for the reversal trials for TI Measurements 7 and 8, where each was based on 10 problems. Note that on the reversal trial $(R)$ the average percentage correct was essentially the difference between $100 \%$ and the criterional prevevereal mastery level of $84 \%$. On the average, Ss required $25-30$ trials to achieve criterion, the occasion for cue reversal.

trials-correct counter always registers the number of correct trials out of the last 21 trials. Since trials previous to the last 21 are of no consequence in satisfying the criterion, the memory cell which contained the information from the first of the most recent 21 trials may be appropriated for recording the outcome of each new trial, which, in turn, becomes the 21st trial of the last 21 trials administered. In summary, the trials-correct counter may respond in one of the following ways on any given trial: (1) count up by 1 when a " 0 " is replaced by a " 1 " in any cell; (2) count down by 1 when $a$ " 1 " is replaced by a " 0 " in any cell; and (3) remain unchanged in count if the new information from the current trial is the same as the information already stored in the cell being readdressed.

\section{Random Placement}

The right-left position assignments of the stimuli are determined by a circuit that generates a random sequence, with the single restriction that a stimulus cannot appear in one position more than three times in succession.

\section{Readout}

The front panel of the control unit is equipped with rows of lamps which indicate S's performance per trial and a trials-correct counter displayed by two numerical readout tubes.

The apparatus is equipped with a printout counter and also a data output jack to supply information to a paper tape punch or other recording device so that the data may be automatically collected for further processing by a computer.

\section{Side Preference Detector}

The equipment also has a side-preference detector which stops the test and sounds an alarm should the $S$ make in succession 10 responses to the same position. The $\mathbf{E}$ can then override the randomizer and place the correct stimulus in the position opposite to the one to which the $S$ has been responding, a procedure used in extinguishing side preferences.

\section{REPRESENTATIVE DATA}

Figure 4 portrays representative reversal data obtained subsequent to criterional training in accordance with the $84 \%$ mastery schedule (Table 1) with the test unit. Consistent with earlier report of $T$ data manually collected (Rumbaugh, 1970) and critical to the interests of making defensible comparisons between the reversal performances of species that might differ in mean numbers of trials to achieve the prereversal criterional performance, correlations between trials to criterion and reversal performance were either low or nonsignificant over the course of eight successive TI measurements.

\section{REFERENCES}

DAVENPORT, J. W., CHAMOVE, A. S., \& HARLOW, H. F. The semiautomatic Wisconsin general test apparatus. Behavior Research Methods Instrumentation, 1970, 2, 135-138.

HARLOW. H. F. The formation of learning sets. Psychological Review, 1949, 56, 51-65.

HARLOW, H. F., BROMER, J. A. A test-apparatus for monkeys. Psy chological Record, $1938,2,434$.

MEYER, D, M. TREICHLER, F. R., MEYER, P. M. Discrete-trial training techniques and stimulus variables, In A. M. Schrier, H. F. Harlow, and F. Stollnitz (Eds.), Behavior of nonhuman primates. New York: Academic Press, 1965. Pp. 1-49.

RUMBAUGH, D. M. The transfer index: An alternative measure of learning set Proceedings of the Second International Primate Congress, 1969. Pp. 267-273.

RUMBAUGH, D. M. Learning stills of anthropolds. In L. A. Rosenblum (Ed.), Primate behavior. New York: Academic Press, 1970. Pp. 1.70.

\section{NOTES}

1. Special circuit parts (emiautomatic unit):

Relays: RL 1 and 2 Potter and Brumfield KCP 1410,000 ohms (Allied Electronics) RL 3 and 4 Price Electric-Type 5315 HP (Price Electric Co., Frederic, Md.).

Photocells: Hunter Mfg. Co. Model 31 photocell.

Transformers: Stancor PA 8421 or Knight 6K 28 HF 125 V, 50 mA; 6.8 V, 2 A (Allied Eleetronies). Knight 6K $113 \mathrm{HF}$ or similar $24.0 \mathrm{~V} 1 \mathrm{~A}$.

Tone zeneretor: Cordover Code Practice Oscillator CPO-4 (Burstein Applebee No, 20 A $1177, \$ .98$ )

Sockets: Ji (socket for lamp circuits) Cinch-Jones S-304-AB: J2 (socket for control circuits) Cinch-Jones S-312-AB, Allied Electronics.

Diodes: All diodes 1-A rectifier Type $200 \mathrm{~V}$ or higher (Allied Electronics). Total cost of parts-\$100-\$150.

2. A circuil diagram and description is not included herewith, since the system is fairly complex; however, the complete diagram and cixcuit description is on permanent file with $D$. M. Rumbaugh of the Yerkes Regional Primate Research Center, Emory University, Atlanta, Georgia 30322 , and may be obtained free upon written request to him. Proof of the logic is available also. Cost of equipment: electrical components-750; packaging (cabinets, etc.) $\$ 250$; projectors $-\$ 80$.

3. Appreciation is expressed to Mr. Ronald Jones of Solar for his photorraphic assistance. 